\title{
Pancreas Cancer Precision Treatment Using Avatar Mice from a Bioinformatics Perspective
}

\author{
Javier Perales-Patón ${ }^{\mathrm{a}}$ Elena Piñeiro-Yañez ${ }^{\mathrm{a}}$ Héctor Tejero ${ }^{\mathrm{a}}$ \\ Pedro P. López-Casas ${ }^{b}$ Manuel Hidalgo ${ }^{c}$ Gonzalo Gómez-López ${ }^{a}$ \\ Fátima Al-Shahrour ${ }^{\text {a }}$ \\ a Bioinformatics Unit, and ${ }^{b}$ Clinical Research Programme, Spanish National Cancer Research Centre (CNIO), \\ Madrid, Spain; ' Beth Israel Deaconess Medical Center, Boston, MA, USA
}

\section{Keywords}

Bioinformatics · Drug repositioning · Pancreatic cancer ·

Personalized medicine $\cdot$ Xenografts

\begin{abstract}
Pancreatic ductal adenocarcinoma (PDAC) is a leading cause of cancer-related death among solid malignancies. Unfortunately, PDAC lethality has not substantially decreased over the past 20 years. This aggressiveness is related to the genomic complexity and heterogeneity of PDAC, but also to the absence of an effective screening for the detection of early-stage tumors and a lack of efficient therapeutic options. Therefore, there is an urgent need to improve the arsenal of anti-PDAC drugs for an effective treatment of these patients. Patient-derived xenograft (PDX) mouse models represent a promising strategy to personalize PDAC treatment, offering a bench testing of candidate treatments and helping to select empirical treatments in PDAC patients with no therapeutic targets. Moreover, bioinformatics-based approaches have the potential to offer systematic insights into PDAC etiology predicting puta-
\end{abstract}

\section{KARGER}

(C) 2017 S. Karger AG, Basel

E-Mail karger@karger.com

www.karger.com/phg tively actionable tumor-specific genomic alterations, identifying novel biomarkers and generating disease-associated gene expression signatures. This review focuses on recent efforts to individualize PDAC treatments using PDX models. Additionally, we discuss the current understanding of the PDAC genomic landscape and the putative druggable targets derived from mutational studies. PDAC molecular subclassifications and gene expression profiling studies are reviewed as well. Finally, latest bioinformatics methodologies based on somatic variant detection and prioritization, in silico drug response prediction, and drug repositioning to improve the treatment of advanced PDAC tumors are also covered.

(C) 2017 S. Karger AG, Basel

\section{Introduction}

Pancreatic ductal adenocarcinoma (PDAC) is the 4 th leading cause of cancer-related deaths [1]. With less than $5 \%$ of the 5 -year overall survival rate, the outcomes of patients with pancreatic cancer have not improved sig-

Fátima Al-Shahrour

Bioinformatics Unit, Spanish National Cancer Research Centre (CNIO)

C/Melchor Fernández Almagro, 3

ES-28029 Madrid (Spain)

E-Mail falshahrour@cnio.es 
nificantly during the past 30 years. The lethality of PDAC stems from a late diagnosis and a lack of efficient therapeutic options [2]. The current therapy of advanced PDAC is the administration of adjuvant systemic chemotherapy. Presently, FOLFIRINOX and nab-paclitaxel plus gemcitabine have only shown modest effectiveness in the overall survival of patients [3]. Therefore, there is urgency in improving the treatment of patients with PDAC.

During the last decade, next-generation sequencing (NGS) techniques have transformed our understanding of the molecular alterations that explain the major features of PDAC $[4,5]$. Genomic analyses on large cohorts of tumors have elucidated the mutational landscape of pancreatic cancer. Unfortunately, no good biomarkers of drug response were found, with the exception of a small subset of patients $(1-5 \%)$ that carry inactivation of DNA maintenance genes (sensitive to platinum-based chemotherapies), BRCA (responders to PARP2 inhibitors) or mutations in druggable oncogenes (ERBB2, MET, FGFR1) [6]. Therefore, at the present time, no biomarker to predict treatment response has reached the clinic and patients are treated in a one-size-fits-all approach. Once patients developed disease progression, they are either enrolled in clinical studies with new agents or treated with additional standard chemotherapy regimens. The outcome of patients treated with second-line chemotherapy is poor, with less than $20 \%$ of 1 -year survival [7]. Thus, tailoring therapies in patients with PDAC is still challenging.

\section{Genomic Understanding of PDAC}

Promising advances have been done through the genomic classification of PDAC into molecular classes, which lead to clinical implications in the expected prognosis and drug response for the patients [8]. These stratifications were established based on patterns of genomic structural alterations or gene expression profiles [9-12]. The results of these studies have shown that PDAC is a genetically complex and heterogeneous disease. Subsequent work has shown that this heterogeneity is also present between the primary and metastatic tumors indicating that there is a genetic evolution process from the primary tumor to the fully developed metastatic disease [13, 14]. While most patients had alterations in the TP53, KRAS, p16/CDKN2A and SMAD4 genes, there were a large number of additional mutations in other genes. In general, these mutations alter the functioning of main sig- naling pathways believed to be critical in PDAC genesis and progression.

Gene expression profiles have helped to reveal specific subtypes of pancreatic adenocarcinoma and their clinical implications. To date, Bailey et al. [12] have reported the most comprehensive classification using RNA expression analysis with 4 classes: squamous, pancreatic progenitor, immunogenic and aberrantly differentiated endocrine exocrine (ADEX). These genomic studies provided a classification of pancreatic tumors into distinct biologic subgroups, with the goal of discovering robust prognostic and predictive biomarker signatures to enhance patient outcomes.

It should be noted, however, that (a) these studies have been performed in primary lesions and, as there are data supporting a genetic drift in metastatic lesions, a more comprehensive analysis of metastases would be important, (b) the current data is mostly descriptive with no information regarding the implications of genomic alterations with prognosis and response to treatment, and (c) because most of the studies have been performed in store tissues, there is little functional validation and therapeutic targeting of the discovered abnormalities.

Nowadays, patients with PDAC are treated regardless of the tumor class. The transcriptional dissection of the PDAC classes could lead to the discovery of subtype-specific vulnerabilities to be targeted by drug compounds. However, it is very hard to deduce useful information about targeted therapy from patients with PDAC from genomic alteration profiles by NGS due to the following: (i) recurrent mutations are undruggable (KRAS, TP53, SMAD4, CDKN2A) and (ii) the majority of druggable oncogenes (ERBB2, MET, FGFR1, CDK6, PIK3R3, and PIK3CA), which are mutated at very low prevalence, are activated in PDAC by amplifications, but whole-exome sequencing is very inefficient to detect this type of alterations.

\section{Integrating Genomic Studies with Patient-Derived Xenografts (Avatar Models) to Individualize Treatment of PDAC}

The use of preclinical models is a core component in every aspect of translational cancer research, ranging from the biologic understanding of the disease to the development of new treatments [15]. Patient-derived xenograft (PDX) mouse models have emerged as an important preclinical platform to test drug efficacy, functional assays, to elucidate new treatments and biomarkers in on-
82

Public Health Genomics 2017;20:81-91 DOI: $10.1159 / 000479812$
Perales-Patón et al. 
cology as well as to address clinically relevant questions [16]. International initiatives such as the EuroPDX consortium have emerged with the objectives of creating a uniquely extensive collection of characterized PDX models, to provide a platform for basic research and preclinical studies for the scientific community using PDX models, and to harmonize working practices [17].

Several research laboratories have focused on the generation of PDX (also known as "avatar models") for translational research in PDAC $[18,19]$. The main goals of these projects were to generate a platform of well-characterized models of PDAC for drug screening and biomarker development. These PDX models recapitulate important characteristics of the patient's PDAC environment (drug diffusion, host body for the development of metastasis, same routes of treatment administration, etc.) [20] and in general they can represent tumor heterogeneity by showing different genotypic and phenotypic characteristics (genomic alterations, metastatic capacities, differential drug responses, intratumoral heterogeneity, etc.) that are relevant in the context of the treatment of PDAC. This diversity allows the selection of particular models based on the presence of specific mutations or pathway activation to address different biological hypotheses derived for instance from computational analyses, such as the experimental testing of candidate drugs in a particular PDAC subtype.

Interestingly, avatar models are being used as an in vivo platform to apply the concept of personalized cancer medicine integrating NGS analysis (targeted or wholeexome sequencing) in an extended series of patients to identify putatively actionable tumor-specific genomic alterations and treating them with a battery of anticancer agents and select the most effective one to guide the patient treatment at the time of progression [21].

This strategy represents an excellent approach for personalized medicine and it has been successful in other settings such as breast cancer [22] and lung cancer and melanoma [23]. Nevertheless, it is not straightforward to get all elements aligned and some patients do not benefit for several reasons such as (a) the avatar model does not grow, (b) no targetable alterations or effective agents are found, or (c) the process is too slow and by the time the information is available the patient is not fit to receive treatment anymore [24]. Thus, the two key issues in this kind of project are to take rate of avatar model development and time. Some patients may indeed develop disease progression before the avatar model is available and will have their treatment decision based on the NGS data. In these situations, the implementation of efficient com-

Pancreas Cancer Precision Treatment putational pipelines is essential to address such limitations.

Bioinformatics analyses play a key role to successfully achieve a personalized medicine approach using genomic data and avatar models by identifying druggable targets from NGS data and prioritizing drugs that will be evaluated in the avatar models (Fig. 1). For this purpose, the development of integrative computational analysis of genomics and pharmacogenomics data will allow to integrate large datasets and reduce the complexity of translating information into efficient drug response prediction or biomarker detection. This approach can potentially lead to the discovery of new effective treatments against PDAC, which might not have been found by other conventional approaches.

To identify potential targets and effective drugs from genomics data, three different methodologies can be applied depending on data source and availability: (i) detection of somatic variants, (ii) prioritization of therapeutic vulnerabilities, and (iii) in silico prediction of drug response.

\section{Somatic Variant Detection}

The increasing popularization of NGS studies has led to the necessity of integrated and reliable data analysis pipelines to process the deep-sequencing experiments in an efficient way. This issue is especially relevant in hospitals and research institutes where regular analyses accentuate the demand for intuitive and automated workflows that accelerate the delivery of final results, minimizing human technical error, and ensuring the reproducibility and fidelity of the data obtained.

For this purpose, our group has implemented RUbioSeq, a free multi-platform software suite for the integrated analysis of sequencing experiments [25]. The software has been designed to facilitate routine analysis of wholegenome sequencing in order to improve the reproducibility of the results using automated protocols. Its current release integrates more than 20 well-established tools and supports parallelized and customizable workflows to detect DNA variants, copy number alterations (CNA-seq), epigenetic changes (bisulfite-seq), and DNA-protein binding sites (ChIPseq). Moreover, the latest version of RUbioSeq includes a new graphical user interface, designed for interdisciplinary research groups where bioinformaticians and biomedical researchers work together. The graphical user interface supports two kinds of profiles: (a) a basic user profile where users with limited skills

Public Health Genomics 2017;20:81-91 83 


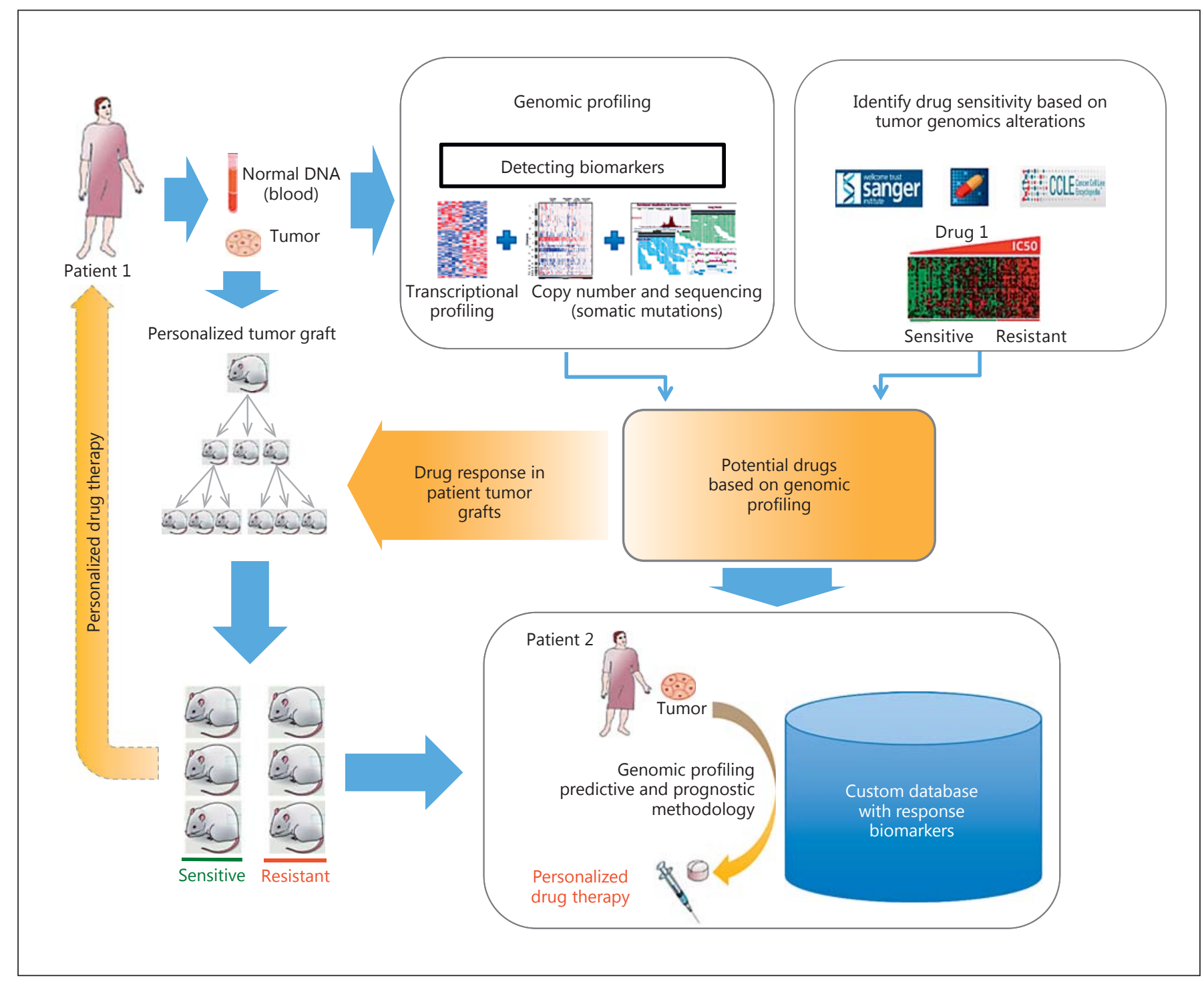

Fig. 1. Representative schema for the treatment prescription of patients with PDAC based on the outcome of tailoring treatments in the patient's personalized tumor graft driven by the identification of targeted tumor vulnerabilities from a retrospective database of drug response biomarkers.

in bioinformatics can execute all the NGS analysis tasks provided by the software and (b) an administration mode where bioinformaticians and advanced users can manage and configure all the technical parameters of the application. Finally, RUbioSeq supports the analysis of matched normal and tumor samples to accurately discern somatic mutations and copy number alterations from germline variants.

On the other hand, some specific difficulties arise when sequencing the genome of PDX samples because host (mouse) material (i.e. DNA/RNA) will inevitably be mixed with the graft (human) material. In such a situation, it is critical to efficiently remove contaminant murine sequence reads from the raw data for the detection of true variants. Computational efforts like the Xenome tool [26] provides a methodology for the specific classification of xenograft-derived sequence read data. Additionally, Tso et al. [27] have indicated that filtering and combined reference strategies performed better than aligning reads directly to human reference in terms of alignment and variant calling accuracies.
Perales-Patón et al. 


\section{Prioritizing Therapeutic Vulnerabilities}

High-throughput sequencing experiments generate lists of hundreds (or thousands) of somatic mutations and other alterations in the same tumor; therefore, we need approaches to establish the relevance of these changes, and more importantly, to prioritize those that could be clinically useful for cancer therapy. A conceivable approach is to prioritize genomic alterations based on tumor-specific vulnerabilities, that is, an altered gene and/ or pathways that drive and maintain tumor cells that often become "addicted" to its deregulated activity and whose inhibition is lethal in cancer cells. Thus, cancer cells rely on the expression of this limited number of specific genes for their survival, and these vulnerabilities are programmed by the underlying molecular features of tumors. Identifying the relationships between these molecular vulnerabilities will yield a comprehensive catalog of the potential therapeutic targets for cancer and provide a rationale for patient stratification.

Several large-scale efforts to detect tumor-specific vulnerabilities include the development of a collection of over 1,000 cancer cell lines with comprehensive molecular and pharmacological characterization, using Genomics of Drugs Sensitivity in Cancer (GDSC) [28], Cancer Cell Line Encyclopedia (CCLE) [29], Cancer Therapeutic Response Portal (CTRP) [30] as well as the systematic use of RNA interference to identify Achilles' heels of cancers, i.e., the Project Achilles [31] and more recently LINCS L1000 resource [32], an extension of CMAP [33].

Nevertheless, none of these resources and methodologies allows automatically relating genomic alterations with matched therapies and candidate drugs for treatment. Tools such as DGIdb [34], CancerResource [35], and the Cancer Genome Interpreter [36] have been developed to identify therapeutically actionable genomic alterations in tumors. Although these tools demonstrate the potential of targeted therapies and provide drug-repurposing strategies, they present some clear limitations including: (i) only known cancer driver genes are considered for drug prescription and based exclusively on somatic DNA alterations, (ii) therapeutic options are restricted to "one target - one drug" ignoring multiple actionable mutations, the protein pathway's context-specific activity, protein-protein interactions and/or regulatory circuits, and (iii) they lack experimental or clinical validation data.

Our group has developed PanDrugs (http://www.pandrugs.org/) with the aim of prioritizing gene variants to guide the selection of therapies from the results of ge-

Pancreas Cancer Precision Treatment nome-wide studies in cancer disease. PanDrugs is a new computational method which overcomes the aforementioned limitations using an intuitive approach: starting from a user-supplied list of genomic alterations or genes, our method automatically annotates them using different sources and calculates a gene score (GScore) based on the level of evidence supporting its actionability considering: (i) genomic feature evidence by mutation consequence, functional impact and pathogenicity, (ii) gene relevance in cancer, (iii) gene essentiality from RNA interference experiments in cancer cell lines, and (iv) clinical implications [37]. Then PanDrugs interrogates its database (PanDrugsdb) to evaluate the therapeutic implications of the precomputed variants or genes and it calculates a drug score (DScore) which takes into account: (i) drug clinical status (approved by the FDA, clinical trials or preclinical), (ii) drug-disease indication, (iii) drug-gene association (direct target, pathway member or biomarker) and number of databases supporting this association, (iv) number of alterations (individual or sets) and their collective impact in relation to the treatment, and (v) pharmacological information retrieved from the literature manually $\mathrm{cu}-$ rated by experts. Finally, PanDrugs intersects GScore and DScore providing a prioritized list of best-candidate therapies that considers the biological relevance of the altered genes in cancer, their therapeutic vulnerability and the clinical application of the drugs (Fig. 2). The current version of PanDrugs integrates data from 18 primary sources and supports $>50 \mathrm{~K}$ drug-target associations obtained from $\sim 6,000$ genes and $\sim 11,000$ unique compounds and supports an API allowing developers to integrate the tool within their own algorithms and NGS analysis pipelines. We think that this tool might provide a feasible approach to guide genomic hypothesis therapies as well as to prioritize multiple actionable mutations in genomically complex tumors.

Several contributions have been carried out in pancreatic cancer to prioritize tumor vulnerabilities. In all these studies, bioinformatics analyses had an essential role. For instance, Biankin et al. [5] performed exome sequencing and copy number analysis in 142 PDAC patients uncovering novel and significantly mutated genes involved in chromatin modification (EPC1 and ARID2), DNA damage repair (ATM), and other mechanisms (ZIM2, MAP2K4, NALCN, SLC16A4, and MAGEA6). More recently, Waddell et al. [6] performed whole-genome sequencing and $\mathrm{CNV}$ analysis of 100 PDACs, proposing new candidate drivers of pancreatic carcinogenesis (KDM6A and PREX2) and classifying PDACs into 4 subtypes with potential clinical utility based on patterns of

Public Health Genomics 2017;20:81-91 DOI: $10.1159 / 000479812$ 


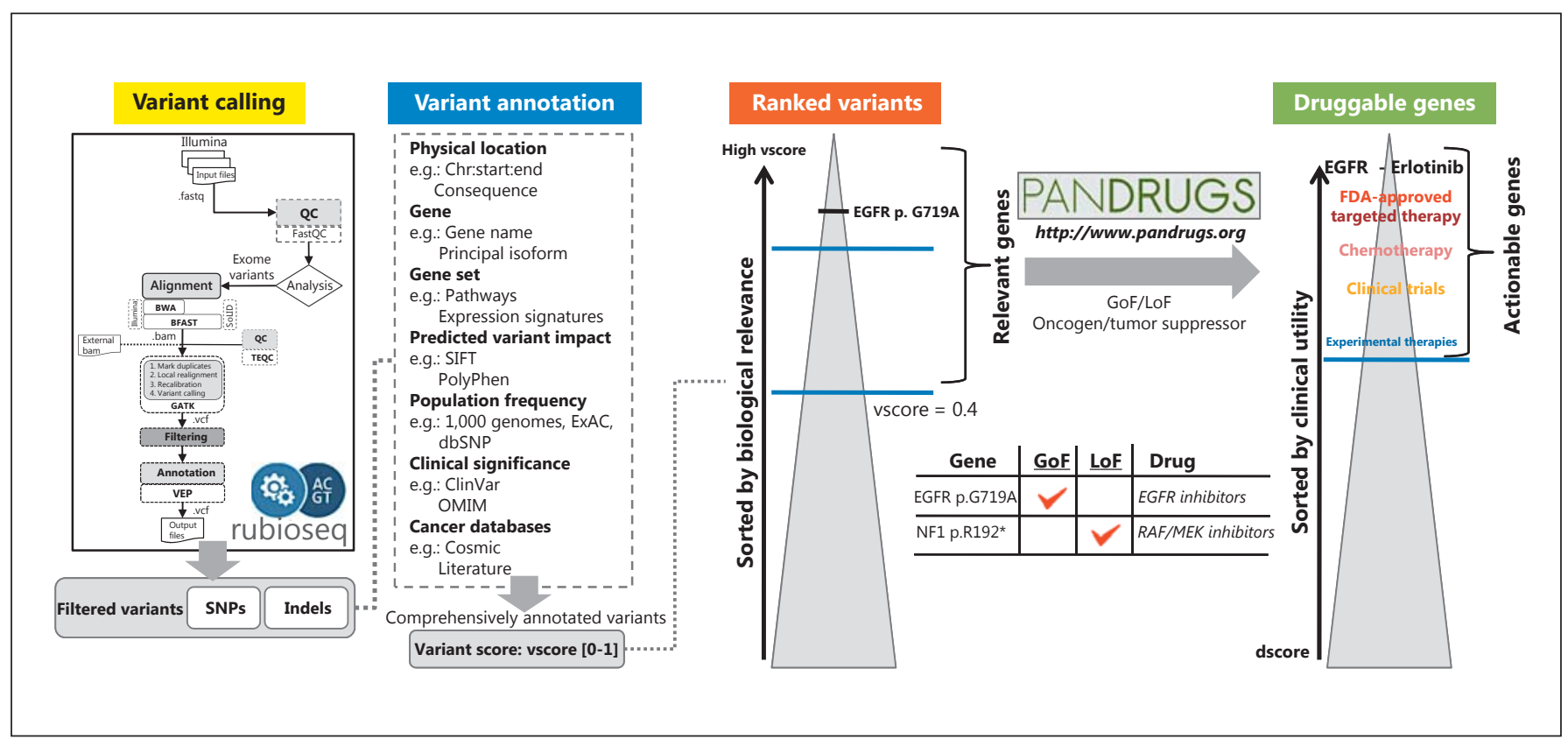

Fig. 2. Workflow for the identification and prioritization of $d r u g$ gable cancer genes from patient's genomic profiling data. Variant calling is performed using RubioSeq [25], a bioinformatics pipeline of gold standard methods that is able to discern somatic mutations from DNA-sequencing data derived from the patient's tumor and matched normal samples. Next, filtered variants (somatic mutations and relevant germinal variants) are fully annotated by integrating different databases and computational resources related to the consequence and functional impact of the variant (based on predictions of deleteriousness and records of the clinical significance), the global frequency in cancer cell lines and healthy human population, and the role in cancer of the particular gene affected by the variant (oncogene or tumor suppressor). Annotated variants are prioritized by weighting the different annotations related to the pathogenicity of the disease. Targeted therapies on biological relevant genes affected by these severe variants are obtained by querying PanDrugs (http://www.pandrugs.org). structural variation. The study also showed a significant proportion of harbored focal amplifications, many of which contained druggable oncogenes (ERBB2, MET, FGFR1, CDK6, PIK3R3, and PIK3CA), but at low individual patient prevalence.

Other studies highlighted the role of genomic profiling using a commercially available platform in patients with advanced cancer. Dhir et al. [38] suggested that this approach is feasible as $97 \%$ of the samples could be successfully amplified and the median time to reporting was 11 days. Recommendations for therapy were new and clinically relevant in $38 \%$ of the patients, but only $13.7 \%$ eventually received genomic-guided therapy. The majority of these patients had advanced disease and a rapid clinical decline that prevented them from receiving genomicguided therapy off-label or enrollment in an appropriate clinical trial. Garralda et al. [39] performed a pilot project to integrate avatar mouse models and genomic analysis in an extended series of patients. In that study, the authors performed whole-exome sequencing analysis of 25 pa- tients with advanced solid tumors to identify putatively actionable tumor-specific genomic alterations. Avatar models were used as an in vivo platform to test proposed treatment strategies. Successful exome sequencing analyses have been obtained for 23 patients. Tumor-specific mutations and copy number variations were identified in all samples. Tumors were implanted to create an avatar model from 14 patients and 10 succeeded. Occasionally, actionable alterations such as mutations in NF1, PI3KA, and DDR2 failed to provide any benefit when a targeted drug was tested in the avatar and, accordingly, treatment of the patients with these drugs was not effective. Thirteen patients received a personalized treatment and 6 achieved durable partial remissions. Prior testing of candidate treatments in avatar models correlated with clinical response and helped to select empirical treatments in some patients with no actionable mutations. These studies suggest that undertaking genomic tumor profiling earlier in a patient's disease course may facilitate the identification and utility of directed protocol therapies.
Perales-Patón et al. 


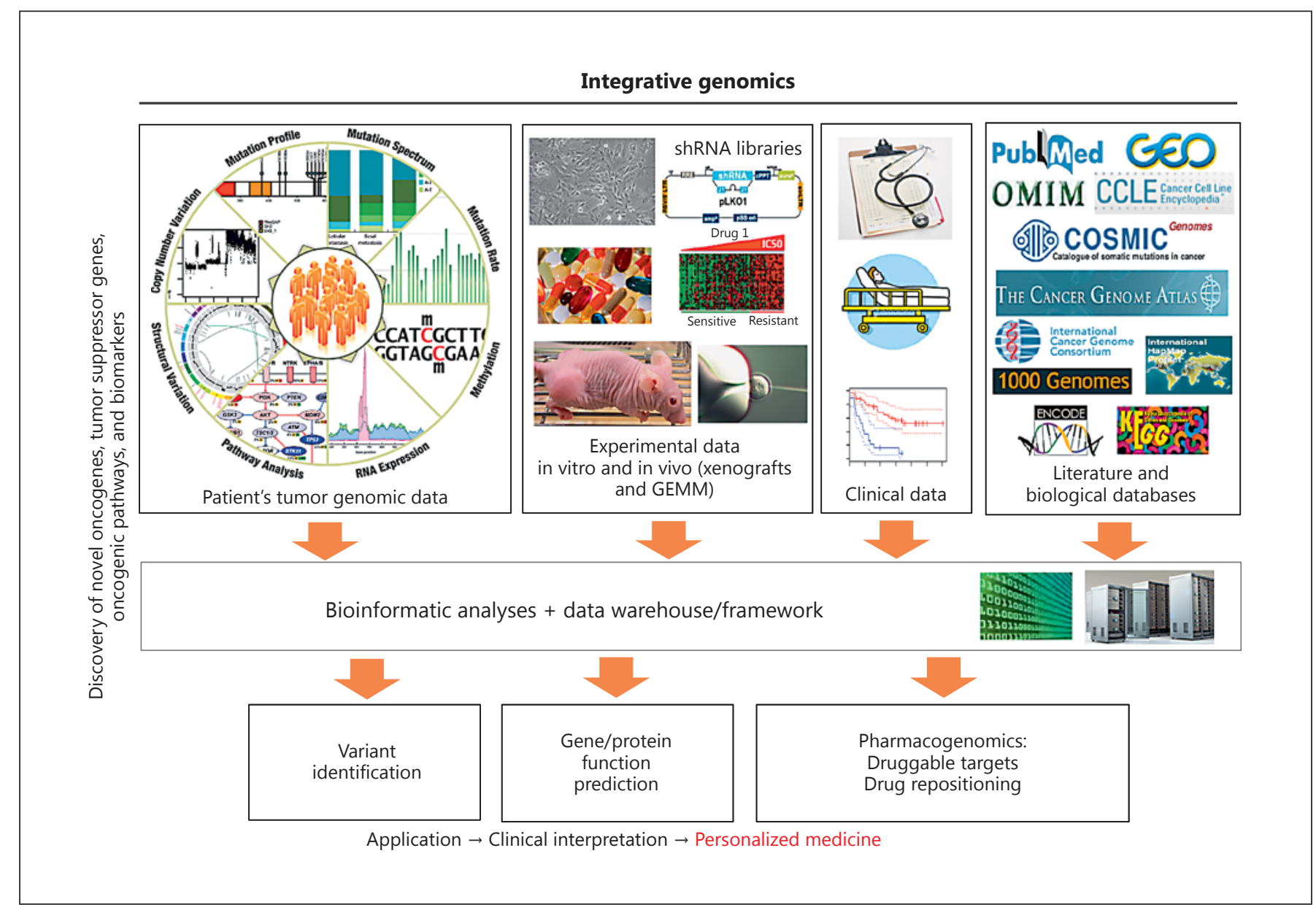

Fig. 3. The new challenge in translational bioinformatics is the integration of a variety of data resources for the understanding of the molecular factors driving tumorigenesis and the discovery of novel associations of drug response in an individualized basis.

All these studies have employed bioinformatics tools and resources proposing new gene-drug response associations and genetic vulnerabilities providing new opportunities to expand the list of pharmacogenomics biomarkers, as well as the design of clinical trials of investigational compounds being developed for the clinic. However, to achieve this purpose, most of the developed strategies have focused on finding only genetic vulnerability associations with a limited resolution and ignoring additional molecular levels, thus, new integrative genomic approaches including genetic, epigenetic and transcriptional data are required (Fig. 3). Promising strategies include the application of drug repositioning approach data to identify new effective drug candidates and predictive biomarkers as well as to predict drug response using patients' tumor gene expression profiles.

Pancreas Cancer Precision Treatment

\section{Drug Repositioning}

Drug repositioning or drug repurposing aims to find new therapeutic indications of established drug compounds [40], avoiding early phases of the drug development process and accelerating their clinical impact in patients who will benefit from a potential new therapy sooner [41]. Drug repositioning activity is based on the fact that a single molecule can have different targets and its modulating effect may depend on the cell context. One popular approach is based on the connectivity map concept. The connectivity map has the potential to provide systematic insights into the complex relationships amongst drugs, genes, and diseases. By examining drugs whose gene expression signatures are opposite to the transcriptional changes of the biological state of interest

Public Health Genomics 2017;20:81-91 DOI: $10.1159 / 000479812$ 
(e.g., a disease), candidate drugs can be proposed for the treatment of the disease [42].

In cancer, different studies have shown that by using drug libraries [43] or by computational approaches [44], new antitumor activities have been found for known drugs already used for other diseases. On the contrary, cancer presents several advantages for drug repositioning due to the great abundance of genomic data coming from particular studies (stored in public repositories) or from the big international projects of molecular character cancer such as TCGA and ICGC [45].

Drug reposition techniques include the use of gene expression signatures (GES) [46], in this case as molecular features representing the transcriptional changes caused by drug exposure. These GES can be used for drug repositioning either by looking for drugs with similar signatures or, following the "signature reversion" paradigm, by looking for drugs with an opposite signature for a given disease or phenotype [47]. The connectivity map was a pioneer approach to test this concept, in which the effect on gene expression of 130,464 drugs was studied in 3 cell lines. Using this resource, several examples of drug repositioning have been proposed as the use of an anticonvulsant against inflammatory bowel disease [48]. Recently, the NIH has been generating and making publicly available the library of integrated network-based cellular signatures, LINCS, in which the GES of about 20,500 small molecules ( 1,300 FDA approved) has been studied in more than 50 cancer cell lines, as well as the GES associated with the silencing using shRNA or overexpression of more than 2,000 genes. The resulting profiles are suitable for molecular signature generation, querying of signatures across cell perturbations and types, and modelling of response networks in cellular systems. Another approach for drug repositioning is the development of network-based models of the disease in order to find potentially relevant targets that could be pharmacologically actionable [49]. Transcription networks [50], Boolean networks [51], and genome-scale metabolic networks [52] can be built from genomic data and can then be used to study the effect of genetic or drug perturbations on the networks.

On the other hand, system biology approaches create an analytical environment to dissect the complexity of the transcriptome signaling of the tumor. Gene regulatory network analysis is capable of elucidating master regulator proteins involved in bottlenecks of the transcriptional programs [53]. Theoretically, these master regulator proteins play a central role in the transcriptional architecture of the tumor by canalizing the upstream effects of onco- genic driver mutations and other aberrant signals to the rest of the network downstream. Targeted therapies against these master regulator proteins could lead to an efficient treatment of the disease $[54,55]$.

Additional studies to identify gene expression and metabolomics biomarkers for patient selection are necessary for the strategic deployment of drugs in the treatment of pancreatic cancer. Ma et al. [56] developed a combined approach to predict candidate drugs for treating pancreatic cancer. The authors first identified potentially useful drugs for pancreatic cancer by using C-Map-based gene expression correlation analyses. Then applied Met-express algorithm to predict key pancreatic cancer enzymes involved in pancreatic cancer metabolism. Finally, they selected candidates from the potentially useful drugs by requiring that their targets be key pancreatic cancer enzymes or the substrates/products of key pancreatic cancer enzymes. Using this approach, the authors predicted 7 candidate drugs for treating pancreatic cancer, 3 of which are supported by literature evidence, and 3 were experimentally validated to be inhibitory to pancreatic cancer cell lines.

Other approaches include the use of GES as predictive biomarkers of drug response. PDXs are an excellent preclinical model to test drug efficacy and their response pattern could be correlated with genomic status as well as transcriptional signatures. To evaluate this approach, we have evaluated the activity of some metabolic inhibitors in pancreatic cancer PDXs. Using a phenformin-induced transcriptomic signature from the LINCS database (http://www.lincsproject.org), we found a correlation between baseline expressions of the signature with sensitivity to phenformin [57]. Although a recent clinical study of metformin failed to demonstrate efficacy [58], this study suggests that this drug could be revived for clinical use and that additional studies to identify gene expression and metabolomics biomarkers for patient selection are necessary for the strategic treatment of pancreatic cancer.

\section{Perspectives}

Developing new therapeutic drugs for PDAC is an urgent need. In this article, we have shown that genomic data analysis is a powerful approach for personalized cancer treatment and coupled with the use of PDX models provides a strategic platform to experimentally test treatment opportunities emanating from the analysis of the cancer genome.

PDX models are becoming an integral part of drug development, including drug screening and biomarker dis-
88

Public Health Genomics 2017;20:81-91 DOI: $10.1159 / 000479812$
Perales-Patón et al. 
covery. International efforts such as EuroPDX have emerged to harmonize and standardize study design and data analysis.

In addition, PDX models bear the promise of assisting clinical trial designs as well as being integrated into personalized medicine strategies. For this last goal, we have presented some pioneer studies that have evaluated this approach by testing the integration of NGS data analysis with PDX models. These studies provide the first steps to promote co-clinical trials, within which patients and PDXs reciprocally inform therapeutic decisions.

A relevant characteristic of the PDX models for personalized medicine is the ability of predicting clinical outcomes which is being improved through mouse humanization strategies. Humanized mice are immunocompromised mice in which selected immune components have been introduced to generate a competent human immune system with different degrees of immune reconstitution. The development of a personalized immune mouse with a robust immune reconstitution with hematopoietic stem cells from the bone marrow of an individual patient may provide a new model to evaluate cancer immunotherapies.

Immunotherapy arises as an alternative and promising strategy for PDAC clinical management and treatment. Knudsen et al. [59] have recently shown the relationship amongst mutational burden of PDAC and the immunosuppressive mechanisms that are conditioned by the tumor stromal environment. The authors observed that PDAC cases exhibited distinct stromal phenotypes that were associated with prognosis, glycolytic and hypoxic biomarkers and immune infiltrate composition and have proposed novel PDAC subtypes for utilizing immunotherapy in the treatment of PDAC. A complementary strategy is to repress protumoral signals induced by immunological tumor-infiltrated cells in PDACs. For instance, macrophages and myeloid-derived suppressor cells not only mediate immune suppression, but also promote metastatic dissemination and impart resistance to cytotoxic therapies. In this sense, Zhu et al. [60] have employed mouse models to show that the inhibition of signaling by the myeloid growth factor receptor CSF1R can functionally reprogram macrophage responses that enhance antigen presentation and productive antitumor Tcell responses. In this study, the authors show that PD1 and CTLA4 antagonists combined with CSF1R blockade potently elicited tumor regressions, even in larger established tumors empowering the therapeutic effects of checkpoint-based immunotherapeutics. New promising computational approaches begin to appear for drug repo-

Pancreas Cancer Precision Treatment sitioning on the immune system showing their potential applicability in cancer. For instance Kidd et al. [61] have modelled the interactions between 1,309 drugs and 221 immune cell types and predict 69,995 interactions providing an immune-cell pharmacology map to predict how 5 drugs influence 4 immune cell types in humans and mice.

However, the application of this approach to clinical practice remains daunting due to a lack of predictive genomic biomarkers implemented in the clinical settings (actually there are only few molecular predictors of efficacy for cancer drugs approved by the FDA [62]), the difficulty to modify established clinical modalities, the overall need to improve the clinical trial design, technological and human resources to accomplish these many tasks, and all in a challenging financial environment.

Pharmacogenomic studies (i.e., CCLE, GDSC, CTRP, and LINCS) have proposed new gene-drug response associations and genetic vulnerabilities providing new opportunities to expand the list of pharmacogenomics biomarkers as well as the design of clinical trials of investigational compounds being developed for the clinic. More specifically, the C-Map and LINCS projects have provided a wealth of gene expression data that can be mined for repositioning drugs, a promising approach to new drug discovery. To exploit these resources will involve the development of more sophisticated computational tools that will have the ability to expand the therapeutic options by predicting candidate targets as well as providing drug mechanisms of action.

Precision medicine will revolutionize health care and will play a dominant role in the future of cancer therapy. Over the next several years, it may indeed be possible to identify patients who will benefit from treatment based on their molecular profile, and to tailor therapeutic regimens accordingly. This will contribute to the sustainability of health and care systems. However, progress overall has been slow despite considerable effort and investment, and there are still many challenges and obstacles to overcome before personalized medicine can be systematically used.

We believe that an integrative strategy using personalized PDX models and genomics data analysis will help to establish new perspectives on personalized cancer treatment and will facilitate the development of genomicbased clinical tests to help categorize tumors and match them to effective drugs or treatments. The strength of this approach lies in the combination of clinical research, genomics, and latest in silico (computational) analyses.

Public Health Genomics 2017;20:81-91 DOI: $10.1159 / 000479812$ 


\section{Acknowledgements}

We thank Dr. J.M. Fernández for critically reading the manuscript. Part of our own work has been supported by the MarieCurie Career Integration Grant (CIG) CIG334361. J.P.-P. is a recipient of a Severo Ochoa fellowship by the Spanish Ministry of Economy and Competitiveness.

\section{Disclosure Statement}

The authors declare that they have no relevant or material financial interests that relate to the research described in this paper.

\section{References}

1 Ryan DP, Hong TS, Bardeesy N: Pancreatic adenocarcinoma. N Engl J Med 2014;371: 1039-1049.

2 Garrido-Laguna, I, Hidalgo M: Pancreatic cancer: from state-of-the-art treatments to promising novel therapies. Nat Rev Clin Oncol 2015;12:319-334.

-3 Von Hoff DD, Ervin T, Arena FP, Chiorean EG, Infante J, Moore M, Seay T, Tjulandin SA, Ma WW, Saleh MN, Harris M, Reni M, Dowden S, Laheru D, Bahary N, Ramanathan RK, Tabernero J, Hidalgo M, Goldstein D, Van Cutsem E, Wei X, Iglesias J, Renschler MF: Increased survival in pancreatic cancer with nab-paclitaxel plus gemcitabine. $\mathrm{N}$ Engl J Med 2013;369:1691-1703.

4 Jones S, Zhang X, Parsons DW, Lin JC, Leary $\mathrm{RJ}$, Angenendt $\mathrm{P}$, et al: Core signaling pathways in human pancreatic cancers revealed by global genomic analyses. Science 2008;321: 1801-1806.

5 Biankin AV, Waddell N, Kassahn KS, Gingras MC, Muthuswamy LB, Johns AL, et al: Pancreatic cancer genomes reveal aberrations in axon guidance pathway genes. Nature 2012; 491:399-405.

-6 Waddell N, Pajic M, Patch AM, Chang DK, Kassahn KS, Bailey P, et al: Whole genomes redefine the mutational landscape of pancreatic cancer. Nature 2015;518:495-501.

-7 Xiong HQ, Varadhachary GR, Blais JC, Hess KR, Abbruzzese JL, Wolff RA: Phase 2 trial of oxaliplatin plus capecitabine (XELOX) as second-line therapy for patients with advanced pancreatic cancer. Cancer 2008;113:20462052.

-8 Pishvaian MJ, Brody JR: Therapeutic implications of molecular subtyping for pancreatic cancer. Oncology (Williston Park) 2017;31: 159-166, 168.

9 Badea L, Herlea V, Dima SO, Dumitrascu T, Popescu I: Combined gene expression analysis of whole-tissue and microdissected pancreatic ductal adenocarcinoma identifies genes specifically overexpressed in tumor epithelia. Hepatogastroenterology 2008; 55: 2016-2027.

10 Collisson EA, Sadanandam A, Olson P, Gibb WJ, Truitt M, Gu S, Cooc J, Weinkle J, Kim GE, Jakkula L, Feiler HS, Ko AH, Olshen AB, Danenberg KL, Tempero MA, Spellman PT, Hanahan D, Gray JW: Subtypes of pancreatic ductal adenocarcinoma and their differing re- sponses to therapy. Nat Med 2011;17:500503.

-11 Moffitt RA, Marayati R, Flate EL, Volmar KE, Loeza SG, Hoadley KA, Rashid NU, Williams LA, Eaton SC, Chung AH, Smyla JK, Anderson JM, Kim HJ, Bentrem DJ, Talamonti MS, Iacobuzio-Donahue CA, Hollingsworth MA, Yeh JJ: Virtual microdissection identifies distinct tumor- and stroma-specific subtypes of pancreatic ductal adenocarcinoma. Nat Genet 2015;47:1168-1178.

12 Bailey P, Chang DK, Nones K, Johns AL, Patch AM, Gingras MC, et al: Genomic analyses identify molecular subtypes of pancreatic cancer. Nature 2016;531:47-52.

13 Campbell PJ, Yachida S, Mudie LJ, Stephens PJ, Pleasance ED, Stebbings LA, et al: The patterns and dynamics of genomic instability in metastatic pancreatic cancer. Nature 2010; 467:1109-1113.

14 Yachida S, Jones S, Bozic I, Antal T, Leary R, Fu B, Kamiyama M, Hruban RH, Eshleman JR, Nowak MA, Velculescu VE, Kinzler KW, Vogelstein B, Iacobuzio-Donahue CA: Distant metastasis occurs late during the genetic evolution of pancreatic cancer. Nature 2010; 467:1114-1117.

15 Hidalgo M, Amant F, Biankin AV, Budinská E, Byrne AT, Caldas C, Clarke RB, de Jong S, Jonkers J, Mælandsmo GM, Roman-Roman S, Seoane J, Trusolino L, Villanueva A: Patient-derived xenograft models: an emerging platform for translational cancer research. Cancer Discov 2014;4:998-1013.

-16 Tentler JJ, Tan AC, Weekes CD, Jimeno A, Leong S, Pitts TM, Arcaroli JJ, Messersmith WA, Eckhardt SG: Patient-derived tumour xenografts as models for oncology drug development. Nat Rev Clin Oncol 2012;9:338-350.

17 Byrne AT, Alférez DG, Amant F, Annibali D, Arribas J, Biankin AV, et al: Interrogating open issues in cancer precision medicine with patient-derived xenografts. Nat Rev Cancer 2017;17:254-268.

18 Garrido-Laguna I, Uson M, Rajeshkumar NV, Tan AC, de Oliveira E, Karikari C, Villaroel MC, Salomon A, Taylor G, Sharma R, Hruban RH, Maitra A, Laheru D, RubioViqueira B, Jimeno A, Hidalgo M: Tumor engraftment in nude mice and enrichment in stroma-related gene pathways predict poor survival and resistance to gemcitabine in patients with pancreatic cancer. Clin Cancer Res
2011;17:5793-5800.

19 Rubio-Viqueira B, Jimeno A, Cusatis G, Zhang X, Iacobuzio-Donahue C, Karikari C, Shi C, Danenberg K, Danenberg PV, Kuramochi H, Tanaka K, Singh S, Salimi-Moosavi $\mathrm{H}$, Bouraoud N, Amador ML, Altiok S, Kulesza P, Yeo C, Messersmith W, Eshleman J, Hruban RH, Maitra A, Hidalgo M: An in vivo platform for translational drug development in pancreatic cancer. Clin Cancer Res 2006; 12:4652-4661.

20 Kim MP, Evans DB, Wang H, Abbruzzese JL, Fleming JB, Gallick GE: Generation of orthotopic and heterotopic human pancreatic cancer xenografts in immunodeficient mice. Nat Protoc 2009;4:1670-1680.

21 Witkiewicz AK, Balaji U, Eslinger C, McMillan E, Conway W, Posner B, Mills GB, O’Reilly EM, Knudsen ES: Integrated patient-derived models delineate individualized therapeutic vulnerabilities of pancreatic cancer. Cell Rep 2016;16:2017-2031.

22 Bruna A, Rueda OM, Greenwood W, Batra AS, Callari M, Batra RN, et al: A biobank of breast cancer explants with preserved intratumor heterogeneity to screen anticancer compounds. Cell 2016;167:260-274.e22.

23 Dong X, Guan J, English JC, Flint J, Yee J, Evans K, Murray N, Macaulay C, Ng RT, Gout PW, Lam WL, Laskin J, Ling V, Lam S, Wang $Y$ : Patient-derived first generation xenografts of non-small cell lung cancers: promising tools for predicting drug responses for personalized chemotherapy. Clin Cancer Res 2010;16:1442-1451.

24 Hidalgo M, Bruckheimer E, Rajeshkumar NV, Garrido-Laguna I, De Oliveira E, RubioViqueira B, Strawn S, Wick MJ, Martell J, Sidransky D: A pilot clinical study of treatment guided by personalized tumorgrafts in patients with advanced cancer. Mol Cancer Ther 2011;10:1311-1316.

25 Rubio-Camarillo M, Gómez-López G, Fernández JM, Valencia A, Pisano DG: RUbioSeq: a suite of parallelized pipelines to automate exome variation and bisulfite-seq analyses. Bioinformatics 2013;29:16871689.

26 Conway T, Wazny J, Bromage A, Tymms M, Sooraj D, Williams ED, Beresford-Smith B: Xenome - a tool for classifying reads from xenograft samples. Bioinformatics 2012; 28:i172-i178. 
27 Tso KY, Lee SD, Lo KW, Yip KY: Are special read alignment strategies necessary and costeffective when handling sequencing reads from patient-derived tumor xenografts? BMC Genomics 2014;15:1172.

28 Garnett MJ, Edelman EJ, Heidorn SJ, Greenman CD, Dastur A, Lau KW, et al: Systematic identification of genomic markers of drug sensitivity in cancer cells. Nature 2012;483: 570-575.

-29 Barretina J, Caponigro G, Stransky N, Venkatesan K, Margolin AA, Kim S, et al: The Cancer Cell Line Encyclopedia enables predictive modelling of anticancer drug sensitivity. Nature 2012;483:603-607.

- 30 Basu A, Bodycombe NE, Cheah JH, Price EV, Liu K, Schaefer GI, et al: An interactive resource to identify cancer genetic and lineage dependencies targeted by small molecules. Cell 2013;154:1151-1161.

- 31 Cowley GS, Weir BA, Vazquez F, Tamayo P, Scott JA, Rusin S, et al: Parallel genome-scale loss of function screens in 216 cancer cell lines. Sci Data 2014;1:140035.

32 Subramanian A, Narayan R, Corsello SM, Peck DD, Natoli TE, Lu X, et al: A next generation connectivity map: L1000 platform and the first 1,000,000 profiles. bioRxiv. 2017. http://biorxiv.org/content/early/2017/ 05/10/136168.

- 33 Lamb J, Crawford ED, Peck D, Modell JW, Blat IC, Wrobel MJ, Lerner J, Brunet JP, Subramanian A, Ross KN, Reich M, Hieronymus H, Wei G, Armstrong SA, Haggarty SJ, Clemons PA, Wei R, Carr SA, Lander ES, Golub TR: The Connectivity Map: using gene-expression signatures to connect small molecules, genes, and disease. Science 2006;313:1929-1935.

-34 Wagner AH, Coffman AC, Ainscough BJ, Spies NC, Skidmore ZL, Campbell KM, Krysiak K, Pan D, McMichael JF, Eldred JM, Walker JR, Wilson RK, Mardis ER, Griffith M, Griffith OL: DGIdb 2.0: mining clinically relevant drug-gene interactions. Nucleic Acids Res 2016;44:D1036-D1044.

35 Gohlke BO, Nickel J, Otto R, Dunkel M, Preissner R: CancerResource - updated database of cancer-relevant proteins, mutations and interacting drugs. Nucleic Acids Res 2016; 44:D932-D937.

36 https://www.cancergenomeinterpreter.org/.

37 Piñeiro-Yañez E, Reboiro-Jato M, PeralesPatón J, Troulé K, Rodriguez JM, Tejero $\mathrm{H}$, Shimamura T, Carretero J, Valencia A, Gómez-López G, Hidalgo M, Glez-Peña D, AlShahrour F: PanDrugs: prioritizing drug treatment in cancer patients according to individual genomic data, submitted.

-38 Dhir M, Choudry HA, Holtzman MP, Pingpank JF, Ahrendt SA, Zureikat AH, Hogg ME, Bartlett DL, Zeh HJ, Singhi AD, Bahary N: Impact of genomic profiling on the treatment and outcomes of patients with advanced gastrointestinal malignancies. Cancer Med 2017; 6:195-206.

39 Garralda E, Paz K, López-Casas PP, Jones S, Katz A, Kann LM, López-Rios F, Sarno F, AlShahrour F, Vasquez D, Bruckheimer E, Angiuoli SV, Calles A, Diaz LA, Velculescu VE, Valencia A, Sidransky D, Hidalgo M: Integrated next-generation sequencing and avatar mouse models for personalized cancer treatment. Clin Cancer Res 2014;20:2476-2484.

40 Xiong HQ, Varadhachary GR, Blais JC, Hess KR, Abbruzzese JL, Wolff RA: Phase 2 trial of oxaliplatin plus capecitabine (XELOX) as second-line therapy for patients with advanced pancreatic cancer. Cancer 2008;113:20462052.

41 Chong CR, Sullivan DJ Jr: New uses for old drugs. Nature 2007;448:645-646.

42 Würth R, Thellung S, Bajetto A, Mazzanti M, Florio T, Barbieri F: Drug-repositioning opportunities for cancer therapy: novel molecular targets for known compounds. Drug Discov Today 2016;21:190-199.

43 Sirota M, Dudley JT, Kim J, Chiang AP, Morgan AA, Sweet-Cordero A, Sage J, Butte AJ: Discovery and preclinical validation of drug indications using compendia of public gene expression data. Sci Transl Med 2011;3:96ra77.

-44 Moffat JG, Rudolph J, Bailey D: Phenotypic screening in cancer drug discovery - past, present and future. Nat Rev Drug Discov 2014;13:588-602.

45 Jahchan NS, Dudley JT, Mazur PK, Flores N, Yang D, Palmerton A, Zmoos AF, Vaka D, Tran KQ, Zhou M, Krasinska K, Riess JW, Neal JW, Khatri P, Park KS, Butte AJ, Sage J: A drug repositioning approach identifies tricyclic antidepressants as inhibitors of small cell lung cancer and other neuroendocrine tumors. Cancer Discov 2013;3:1364-1377.

46 Li J, Zheng S, Chen B, Butte AJ, Swamidass SJ, $\mathrm{Lu} Z$ : A survey of current trends in computational drug repositioning. Brief Bioinform 2016;17:2-12.

47 Iorio F, Rittman T, Ge H, Menden M, SaezRodriguez J: Transcriptional data: a new gateway to drug repositioning? Drug Discov Today 2013;18:350-357.

48 Dudley JT, Sirota M, Shenoy M, Pai RK, Roedder S, Chiang AP, Morgan AA, Sarwal MM, Pasricha PJ, Butte AJ: Computational repositioning of the anticonvulsant topiramate for inflammatory bowel disease. Sci Transl Med 2011;3:96ra76.

49 Iorio F, Saez-Rodriguez J, di Bernardo D: Network based elucidation of drug response: from modulators to targets. BMC Syst Biol 2013;7:139.

50 Sorrells TR, Johnson AD: Making sense of transcription networks. Cell 2015;161:714723.
Wang RS, Saadatpour A, Albert R: Boolean modeling in systems biology: an overview of methodology and applications. Phys Biol 2012;9:055001.

52 O’Brien EJ, Monk JM, Palsson BO: Using genome-scale models to predict biological capabilities. Cell 2015;161:971-987.

53 Califano A, Alvarez MJ: The recurrent architecture of tumour initiation, progression and drug sensitivity. Nat Rev Cancer 2017;17: 116-113.

54 Giulietti M, Occhipinti G, Principato G, Piva F: Weighted gene co-expression network analysis reveals key genes involved in pancreatic ductal adenocarcinoma development. Cell Oncol (Dordr) 2016;39:379-388.

-55 Alvarez MJ, Shen Y, Giorgi FM, Lachmann A, Ding BB, Ye BH, Califano A: Functional characterization of somatic mutations in cancer using network-based inference of protein activity. Nat Genet 2016;48:838-847.

56 Ma Y, Hu J, Zhang N, Dong X, Li Y, Yang B, Tian W, Wang X: Prediction of candidate drugs for treating pancreatic cancer by using a combined approach. PLoS One 2016; 11:e0149896.

57 Rajeshkumar NV, Yabuuchi S, Pai S, De Oliveira E, Kamphorst JJ, Rabinowitz JD, Tejero H, Al-Shahrour F, Hidalgo M, Maitra A, Dang CV: Treatment of pancreatic cancer patient-derived xenograft panel with metabolic inhibitors reveals efficacy of phenformin. Clin Cancer Res 2017, Epub ahead of print.

58 Kordes S, Pollak MN, Zwinderman AH, Mathot RA, Weterman MJ, Beeker A, et al: Metformin in patients with advanced pancreatic cancer: a double-blind, randomised, placebocontrolled phase 2 trial. Lancet Oncol 2015; 16:839-847.

59 Knudsen E, Vail P, Balaji U, Ngo H, Botros IW, Makarov V, Riaz N, Balachandran VP, Leach SD, Thompson DM, Chan TA, Witkiewicz AK: Stratification of pancreatic ductal adenocarcinoma: combinatorial genetic, stromal, and immunological markers. Clin Cancer Res 2017;23:4429-4440.

60 Zhu Y, Knolhoff BL, Meyer MA, Nywening TM, West BL, Luo J, Wang-Gillam A, Goedegebuure SP, Linehan DC, DeNardo DG: CSF1/CSF1R blockade reprograms tumor-infiltrating macrophages and improves response to T-cell checkpoint immunotherapy in pancreatic cancer models. Cancer Res 2014;74:5057-5069.

61 Kidd BA, Wroblewska A, Boland MR, Agudo J, Merad M, Tatonetti NP, Brown BD, Dudley JT: Mapping the effects of drugs on the immune system. Nat Biotechnol 2016; $34: 47-54$

62 http://www.fda.gov/Drugs/ScienceResearch/ ResearchAreas/Pharmacogenetics/. 